\title{
Spontaneous variations in atrioventricular conduction in pre-excitation
}

\author{
R. Parameswaran, Tohru Ohe, Fred K. Nakhjavan, and Harry Goldberg \\ From the Cardiology Section and WLMR Laboratories, Albert Einstein Medical Center, Philadelphia, Pa, \\ U.S.A.
}

\begin{abstract}
Atrial pacing in a patient with a Wolff-Parkinson-White syndrome pattern showed unusual variability in $A V$ nodal conduction time. Changes in the $A V$ nodal conduction time occurred predominantly at paced rates in excess of 100/min and consisted of abrupt or gradual decreases in the AH interval. Such decreases resulted in unexpected normalization of the QRS pattern. In addition, pacing also revealed evidence of block in the accessory pathway. Such changes in atrioventricular conduction may account for the known variability of the QRS pattern in patients with the WPW syndrome. Further, they may also explain the benign clinical course in this patient.
\end{abstract}

Electrical pacing in conjunction with intracardiac electrography has improved our understanding of the Wolff-Parkinson-White (WPW) syndrome (Wellens, 1975). Using these techniques the electrophysiological properties of the atrioventricular (AV) node and the accessory pathway that predispose to and perpetuate supraventricular tachyarrhythmias can be delineated. They have also helped to explain the known variability of the QRS morphology in patients with this syndrome. Delay in $\mathrm{AV}$ nodal conduction leads to ventricular activation predominantly through the accessory pathway, and results in an increase in the QRS deformity, whereas block in the accessory pathway results in diminution or disappearance of the delta waves and normalization of the QRS complex (Massumi and Vera, 1971). In the patient reported here normalization of the QRS pattern occurred because of an unusual mechanism - namely, an unexpected improvement in AV nodal conduction. Electrophysiological studies in this patient also showed spontaneous variations in conduction through the accessory pathway. In addition to its effects on the QRS morphology, spontaneous variability of conduction through both these pathways may also explain the absence of bouts of tachycardia.

\section{Case report}

A 28-year-old white man was admitted for the investigation of a cardiac murmur. Except for one brief episode of 'palpitations' at the age of 18 , he had been asymptomatic. Cardiac examination was unremarkable except for a short, soft systolic murmur along the left sternal border. A routine electrocardiogram revealed type $A$ WPW pattern. Cardiac catheterization and angiography showed no evidence of congenital or acquired heart disease. Because of the absence of bouts of tachycardia with the WPW pattern electrophysiological studies were performed to determine the functional characteristics of the AV nodal and the accessory pathways. Atrial pacing was carried out with a bipolar electrode catheter positioned high in the right atrium. His bundle electrograms were recorded by the technique described by Scherlag and associates (1969).

With increasing rates of atrial pacing there was a progressive increase in the AV nodal conduction time, as indicated by a prolongation of the AH intervals. Conduction through the accessory pathway contributed increasingly to ventricular activation, resulting in larger delta waves. The response to atrial pacing was thus generally similar to that reported previously (Castellanos et al., 1970). However, interesting changes in the AV nodal conduction pattern occurred during pacing at rates in excess of $100 / \mathrm{min}$, consisting of unexpected decreases of the AH interval ranging up to $85 \mathrm{~ms}$. The reduction in the AH interval was often gradual and progressive over several cycles. Rarely, it was abrupt and confined to one or two cycles (Fig. 1A, B). The improvement in AV nodal conduction resulted in a concomitant reduction in the size of the delta waves, since ventricular activation took place predominantly through the AV node and the His bundle. Thus the QRS complexes became more normal in spite of an unchanged rate and in the absence of block in the accessory pathway. 

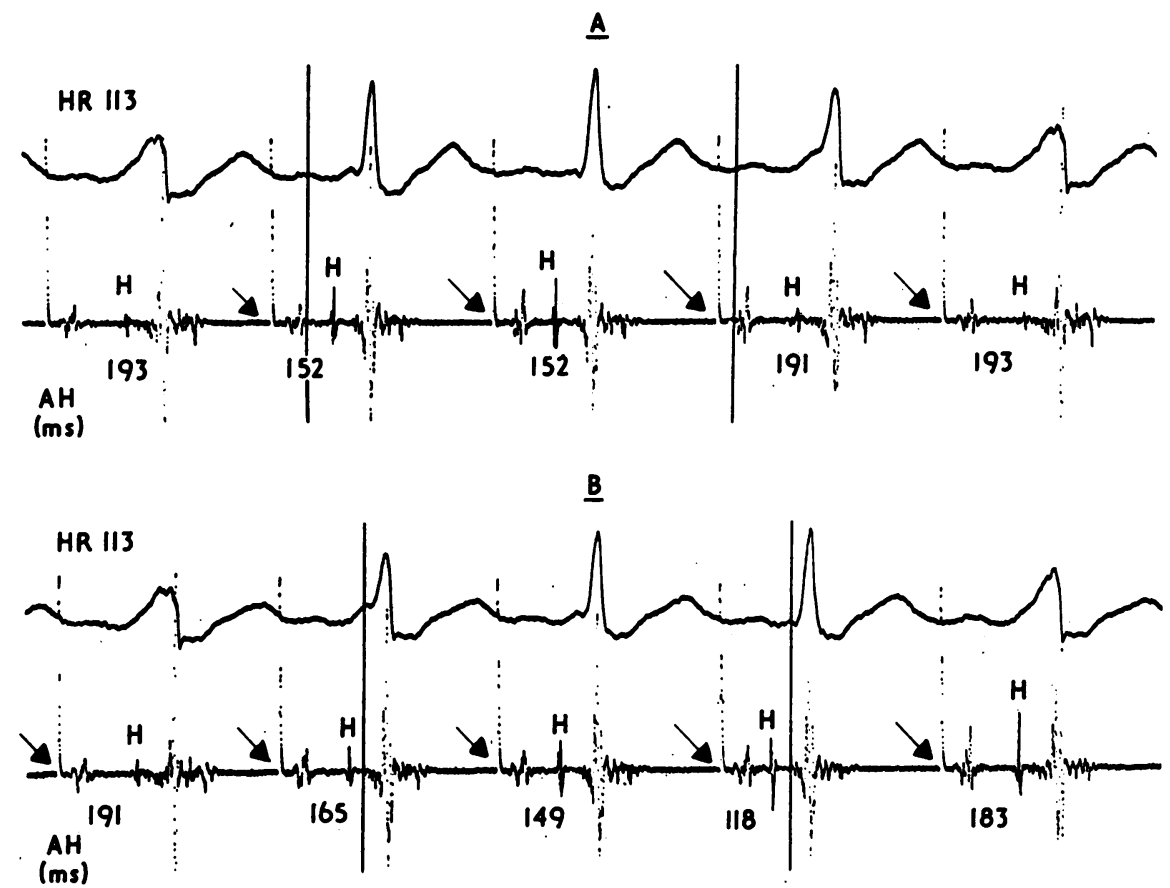

FIG. 1 Representative records made during atrial pacing at 113/min. Arrows indicate stimulus artefacts. Note abrupt decrease in $A H$ interval in second and third beats in panel $A$, and gradual decrease (second, third, and fourth beats) in panel $B$. In both instances improvement in $A V$ nodal conduction results in virtual disappearance of delta wave and normalization of $Q R S$ complex (see text for discussion).

Two additional findings suggested that spontaneous variability of conduction also occurred in the accessory pathway. The first of these was the sudden diminution in the size of the delta wave in isolated beats in the absence of any change in AV nodal conduction (Fig. 2 A), and the second was the observation that block in the accessory pathway induced by pacing was not reproducible. Fig. 2B and $C$ shows an example of this latter phenomenon. At a paced rate of $130 / \mathrm{min}$ refractoriness of the accessory pathway is indicated by the disappearance of the delta waves (Fig. 2B). However, when pacing at the same rate was repeated several minutes later the accessory pathway was no longer refractory, as shown by the reappearance of large delta waves (Fig. 2C). Since these changes in the QRS pattern occurred in the absence of any change in the AH interval they must be attributed to variability of conduction in the accessory pathway.

\section{Discussion}

Of principal interest in the patient reported here is the unusual variability in $\mathrm{AV}$ conduction involving both the AV node and the accessory pathway. Previous reports have suggested that variability in $\mathrm{AV}$ nodal conduction may be the result of spon- taneous changes in the autonomic tone or of functional longitudinal dissociation of the AV node into two or more pathways (Silverman and Goodman, 1951; Kistin, 1963; Langendorf, 1958). The abrupt manner in which the $A H$ intervals decreased and the frequent presence of such a decrease in only one or two beats argue against variations in the autonomic tone being the sole mechanism in our patient. Though functional longitudinal dissociation of the AV node has been shown in patients during atrial pacing (Rosen, Mehta, and Miller, 1974), the applicability of this concept to the patient under discussion is questionable, since the wide range of $\mathrm{AH}$ intervals observed is unusual and would require postulation of several pathways within the AV node. A similar uncertainty exists in attempting to explain the variability of conduction in the accessory pathway. Block in the accessory pathway may occur during premature atrial stimulation and also during rapid atrial pacing (Durrer et al., 1967; Castellanos et al., 1970). Its occurrence in isolated beats, as shown in Fig. 2, and its lack of reproducibility are less common and suggest spontaneous variations in the refractoriness of the accessory pathway. 

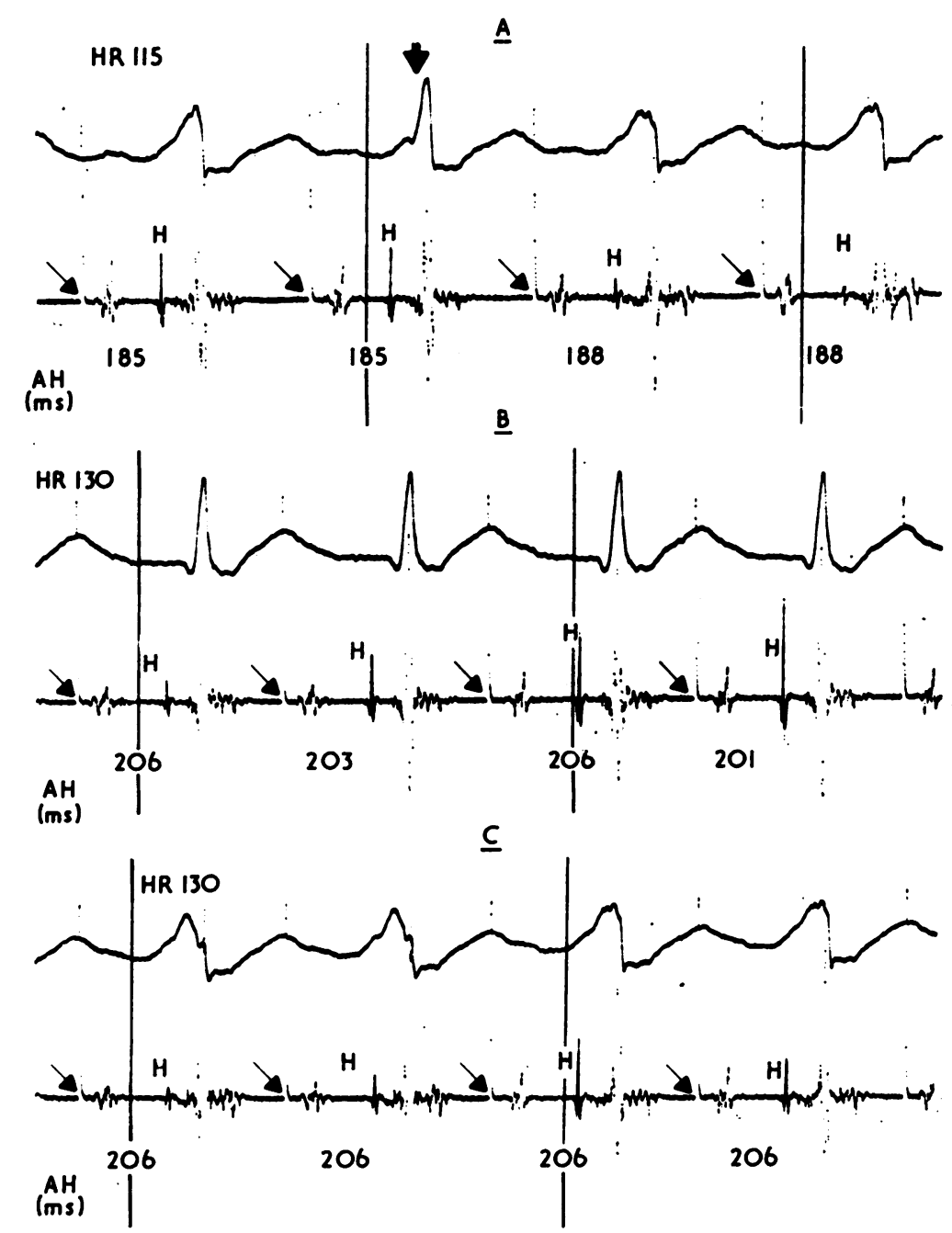

FIG. 2 Panels $A, B$, and $C$ show variability of conduction in accessory pathway. Sudden diminution in size of the delta wave (arrow, panel $A$ ) in absence of any change in $A H$ interval indicates conduction delay in accessory pathway. Panels $B$ and $C$ were recorded during atrial pacing at 130/min. Note absence of delta waves in $B$ and reappearance in $C$ when pacing at same rate was resumed several minutes later (see text for discussion).

Although the mechanisms underlying the variations in atrioventricular conduction are unclear, the effect of such changes is interesting in two respects. The first is its role in the normalization of the QRS complex, and the second is its possible relation to the absence of tachyarrhythmias in this patient. Normalization of the QRS complex occurs because of the development of block in the accessory pathway, but may also be seen in sinus beats terminating long pauses (Massumi and Vera, 1971). In the patient reported here it occurred as the result of an improvement in AV nodal conduction unrelated to a change in rate. The absence of tachycardias noted in this patient agrees with previous clinical observations that in a substantial proportion of patients with the WPW pattern bouts of tachycardia may be short and inconsequential or absent (Chung, Walsh, and Massie, 1965).

The reasons underlying such relative freedom from attacks of tachycardia are not clear. The findings in the patient reported here suggest a possible explanation. The initiation of tachycardia 
in patients with the WPW syndrome depends on a critical disparity in the refractory periods of the AV nodal and accessory pathways. Once initiated, the perpetuation of such tachycardias requires that conduction in the various components of the circuit remains reasonably constant. Significant variations in conduction, abrupt or gradual, as shown in Fig. 1 and 2, may be expected to lead to the cessation of tachycardias. It is reasonable to suggest that a similar variability in the AV nodal and accessory pathway conduction may account for the benign clinical course in other patients with the WPW pattern.

\section{References}

Castellanos, A., Chapunoff, E., Castillo, C., Maytin, O., and Lemberg, L. (1970). His bundle electrograms in two cases of Wolff-Parkinson-White (pre-excitation) syndrome. Circulation, 41, 399.

Chung, K-Y., Walsh, T. J., and Massie, E. (1965). WolffParkinson-White syndrome. American Heart fournal, 69, 116.

Durrer, D., Schoo, L., Schuilenberg, R. M., and Wellens, H. J. J. (1967). The role of premature beats in the initiation and the termination of supraventricular tachycardia in the Wolff-Parkinson-White syndrome. Circulation, 36, 644.
Kistin, A. D. (1963). Multiple pathways of conduction and $\subseteq$ reciprocal rhythm with interpolated ventricular premature systoles. American Heart fournal, 65, 162.

Langendorf, R. (1958). Alternation of A-V conduction time. American Heart fournal, 55, 181.

Massumi, R. A., and Vera, Z. (1971). Patterns and mechanisms of QRS normalization in patients with WolffParkinson-White syndrome. American fournal of Cardio$\log y, 28,541$.

Rosen, K. M., Mehta, A., and Miller, R. A. (1974). Demonstration of dual atrioventricular nodal pathways in man. American fournal of Cardiology, 33, 291.

Scherlag, B. J., Lau, S. H., Helfant, R. H., Berkowitz, W. D., Stein, E., and Damato, A. N. (1969). Catheter technique for recording His bundle activity in man. Circulation, 39, 13.

Silverman, J. J., and Goodman, R. D. (1951). Extraordinary alteration of the P-R interval in neurocirculatory asthenia. American Heart fournal, 41, 155.

Wellens, H. J. J. (1975). Contribution of cardiac pacing to our understanding of the Wolff-Parkinson-White syndrome. British Heart fournal, 37, 231.

Requests for reprints to Dr. R. Parameswaran, Albert Einstein Medical Center, York and Tabor Roads, Philadelphia, Pennsylvania 19141, U.S.A. 